\title{
Maximally entangled mixed states and conditional entropies
}

\author{
J. Batle, ${ }^{1}$ M. Casas, ${ }^{1}$ A. Plastino, ${ }^{2,3}$ and A. R. Plastino ${ }^{3,4,5}$ \\ ${ }^{1}$ Departament de Física, Universitat de les Illes Balears and IMEDEA-CSIC, 07122 Palma de Mallorca, Spain \\ ${ }^{2}$ Argentina's National Research Council (CONICET), Casilla de Correo 727, 1900 La Plata, Argentina \\ ${ }^{3}$ Department of Physics, National University La Plata, Casilla de Correo 727, 1900 La Plata, Argentina \\ ${ }^{4}$ Faculty of Astronomy and Geophysics, National University La Plata, Casilla de Correo 727, 1900 La Plata, Argentina \\ ${ }^{5}$ Department of Physics, University of Pretoria, 0002 Pretoria, South Africa
}

(Received 17 September 2004; published 14 February 2005)

\begin{abstract}
The maximally entangled mixed states of Munro et al. [Phys. Rev. A 64, 030302 (2001)] are shown to exhibit interesting features vis á vis conditional entropic measures. The same happens with the Ishizaka and Hiroshima states [Phys. Rev. A 62, 022310 (2000)], whose entanglement degree cannot be increased by acting on them with logic gates. Special types of entangled states that do not violate classical entropic inequalities are seen to exist in the space of two qubits. Special meaning can be assigned to the Munro et al. special participation ratio of 1.8 .
\end{abstract}

DOI: 10.1103/PhysRevA.71.024301

PACS number(s): 03.67.Mn, 89.70.+c

\section{INTRODUCTION}

Entanglement is one of the most fundamental issues of quantum theory [1]. It is a physical resource, like energy, associated with the peculiar nonclassical correlations that are possible between separated quantum systems. Recourse to entanglement is required so as to implement quantum information processes $[2,3]$ such as quantum cryptographic key distribution [4], quantum teleportation [5], superdense coding [6], and quantum computation [7]. Indeed, production of entanglement is a kind of elementary prerequisite for any quantum computation. A state of a composite quantum system is called "entangled" if it cannot be represented as a mixture of factorizable pure states. Otherwise, the state is called separable. The above definition is physically meaningful because entangled states (unlike separable states) cannot be prepared locally by acting on each subsystem individually $[8,9]$. A physically motivated measure of entanglement is provided by the entanglement of formation $E[\rho][10]$, that quantifies the resources needed to create a given entangled state $\rho$. The entanglement of formation for two-qubits systems is given by Wootters' expression [11], $E[\rho]=h(1$ $\left.+\sqrt{1-C^{2}} / 2\right)$, where $h(x)=-x \log _{2} x-(1-x) \log _{2}(1-x)$, and $C$ stands for the concurrence of the two-qubits state $\rho$. The concurrence is given by $C=\max \left(0, \lambda_{1}-\lambda_{2}-\lambda_{3}-\lambda_{4}\right), \lambda_{i}$, $(i=1, \ldots 4)$ being the square roots, in decreasing order, of the eigenvalues of the matrix $\rho \tilde{\rho}$, with $\tilde{\rho}=\left(\sigma_{y} \otimes \sigma_{y}\right) \rho^{*}\left(\sigma_{y} \otimes \sigma_{y}\right)$. The above expression has to be evaluated by recourse to the matrix elements of $\rho$ computed with respect to the product basis. Another meaningful quantity is the fully entangled fraction $F_{E F}$ [12], that determines the range of possible concurrence values for a mixed state: $F_{E F} \leqslant C \leqslant\left(F_{E F}+1\right) / 2$. For an illustration of this last statement, the reader is referred to Fig. 2 of Ref. [12], whose authors investigate the fraction of two-qubits mixed states that can be used in all quantum information processing applications using $F_{E F}$. Still another important quantity is the participation ratio,

$$
R(\rho)=\left[\operatorname{Tr}\left(\rho^{2}\right)\right]^{-1},
$$

is particularly convenient for calculations and can be regarded as a measure of the degree of mixture of a given density matrix [13-15]. It varies from unity for pure states to $N$ for totally mixed states (if $\hat{\rho}$ is represented by an $N \times N$ matrix). It may be interpreted as the effective number of pure states that enter the mixture. If the participation ratio of $\rho$ is high enough, then its partially transposed density matrix is positive, which for $N=4$ amounts to separability for $R \geqslant 3$ $[9,14]$. Notice also that $R$ is invariant under the action of unitary operators.

There are several entropic (or information) measures that can be useful in order to investigate the violation of classical entropic inequalities by quantum entangled states. Among them, the von Neumann measure is important because of its relationship with the thermodynamic entropy, and the participation ratio is particularly convenient both for numerical and analytical calculations [13-15]. The $q$ entropies, which are functions of the quantity $\omega_{q}=\operatorname{Tr}\left(\rho^{q}\right)$, provide one with a whole family of entropic measures. In the limit $q \rightarrow 1$ these measures incorporate von Neumann's as a particular instance. On the other hand, when $q=2$ they are simply related to the participation ratio (1). Most of the applications of $q$ entropies to physics involve either the Rényi or the Tsallis' entropies [16,17], respectively,

$$
S_{q}^{R}=\ln \left(\omega_{q}\right) /(1-q), \quad S_{q}^{T}=\left(1-\omega_{q}\right) /(q-1) .
$$

In the $q=2$-case, $S_{q=2}^{T}$ is often called the linear entropy $\mathcal{S}_{\mathcal{L}}$ [15]. Tsallis' and Rényi's measures are related through $S_{q}^{T}=F\left(S_{q}^{R}\right)$, where the function $F$ is given by $F(x)$ $=\left\{e^{(1-q) x}-1\right\} /(1-q)$. As an immediate consequence, for all nonvanishing values of $q$, Tsallis' measure $S_{q}^{T}$ is a monotonic increasing function of Rényi's measure $S_{q}^{R}$. Considerable attention has been recently paid to a conditional entropic measure based upon Tsallis' functional, and defined as

$$
S_{q}^{T}(A \mid B)=\left\{S_{q}^{T}(A B)-S_{q}^{T}(B)\right\} /\left\{1+(1-q) S_{q}^{T}(B)\right\} .
$$

Here $\rho_{A B}$ designs an arbitrary quantum state of the composite system $A \oplus B$, not necessarily factorizable nor separable, and $\rho_{B}=\operatorname{Tr}_{A}\left(\rho_{A B}\right)$. The conditional $q$-entropy $S_{q}^{T}(B \mid A)$ is defined in a similar way as (3), replacing $\rho_{B}$ by $\rho_{A}=\operatorname{Tr}_{B}\left(\rho_{A B}\right)$. The 
conditional $q$ entropy (3) has been recently studied in connection with the separability of density matrices describing composite quantum systems [18,19]. For separable states (see for instance [20]),

$$
S_{q}^{T}(A \mid B) \geqslant 0, \quad S_{q}^{T}(B \mid A) \geqslant 0 .
$$

On the contrary, there are entangled states that have negative conditional $q$ entropies. That is, for some entangled states one (or both) of the inequalities (4) are not verified. Now, since Tsallis' entropy is a monotonous increasing function of Rényi's, it is plain that (3) has always the same sign as $S_{q}^{R}(A \mid B)=S_{q}^{R}\left(\rho_{A B}\right)-S_{q}^{R}\left(\rho_{B}\right)$. The positivity of either the Tsallis' conditional entropy or the Rényi conditional entropy are known as the classical $q$-entropic inequalities [20].

In practice, one will more often have to deal with mixed states than with pure ones. From the point of view of entanglement exploitation, one should then be interested in maximally entangled mixed states (MEMS) $\rho_{M E M S}$, which are the basic constituents of all quantum communication protocols. The MEMS states have been studied, for example, in Refs. [15,21,22] for the two-qubits instance of two (one qubit-)subsystems $A$ and $B$. For MEMS, the relations between (i) von Neumann's and linear entropies, on the one hand, and (ii) concurrence and von Neumann entropy, on the other, have been exhaustively investigated in [22]. MEMS states have been recently experimentally encountered $[23,24]$. We will focus attention on these kind of states here. MEMS for a given $R$ value have the following appearance in the computational basis $(|00\rangle,|01\rangle,|10\rangle,|11\rangle)[15]$.

$$
\rho_{\text {MEMS }}=\left(\begin{array}{cccc}
g(x) & 0 & 0 & x / 2 \\
0 & 1-2 g(x) & 0 & 0 \\
0 & 0 & 0 & 0 \\
x / 2 & 0 & 0 & g(x)
\end{array}\right),
$$

with $g(x)=1 / 3$ for $0 \leqslant x \leqslant 2 / 3$, and $g(x)=x / 2$ for $2 / 3 \leqslant x$ $\leqslant 1$. The change of the $g(x)$ regime ensues for $R=1.8$. We will reveal below some physical consequences of this regime change. Of great importance also are the mixed states whose entanglement degree cannot be increased by the action of logic gates [21] that, again in the same basis, are given by

$$
\rho_{I H}=\left(\begin{array}{cccc}
p_{2} & 0 & 0 & 0 \\
0 & \frac{p_{3}+p_{1}}{2} & \frac{p_{3}-p_{1}}{2} & 0 \\
0 & \frac{p_{3}-p_{1}}{2} & \frac{p_{3}+p_{1}}{2} & 0 \\
0 & 0 & 0 & p_{4}
\end{array}\right),
$$

whose eigenvalues are the $p_{i} ;(i=1, \ldots, 4)$ and $p_{1} \geqslant p_{2} \geqslant p_{3}$ $\geqslant p_{4}$. We call these states the Ishizaka and Hiroshima (IH) ones and their concurrence reads $C_{I H}=p_{1}-p_{3}-2 \sqrt{p_{2} p_{4}}$, a relation valid for ranks $\leqslant 3$ that has numerical support also if the rank is four [21]. Of course, all MEMS belong to the IH class. Our goal is to uncover interesting correlations between entanglement and mixedness that emerge when we study these states from the viewpoint of conditional entropies.

\section{ENTROPIC INEQUALITIES AND MEMS}

We begin here with the presentation of our results. A few of them are of an analytical nature. For instance, in the case of all states of the forms (6) and/or (5), the partial traces $\rho_{A / B}$ over one of the subsystems $A$ or $B$ are equal, i.e., for the reduced density matrices we have $\rho_{A}=\rho_{B}$, which entails $S_{q}(A \mid B)=S_{q}(B \mid A)$ for both the Rényi and the Tsallis entropies. Notice that this is a particular feature of these states.

As for the form (6), we establish a lower bound to its states' concurrence for a considerable $R$ range (see Fig. 3), namely,

$$
C_{I H ; M i n}=[\sqrt{3 R(4-R)}-R] /(2 R) .
$$

In the case of MEMS, and in the vicinity of $R=1$, we can analytically relate entropic changes with concurrence changes, in the fashion (remember that for MEMS $C$ $\left.\equiv C_{\operatorname{Max}}\right)$

$$
\Delta S_{q}^{R}(A \mid B)=-[2 q /\{\ln (2)(q-1)\}] \Delta C .
$$

The case $q \rightarrow \infty$ is the strongest $q$-entropic criterion [20]. Equation (8) expresses the fact that, for MEMS, small deviations from pure states (for which the $q$-entropic criteria are necessary and sufficient separability conditions) do not change the criteria's validity, that becomes then extended to a class of mixed states.

\section{A. Numerical results}

We will randomly generate states in the space $\mathcal{S}^{(N)}$ of mixed states $\rho(N=4$ in our case). This can be regarded as a product space, $\mathcal{S}^{(N)}=\mathcal{P} \times \Delta$, where $\mathcal{P}$ stands for the set of orthonormal projectors $\left(\sum_{i=1}^{N} \hat{P}_{i}=I\right)$ and $\Delta$ is the set of all real $N$-uples $\left(\left\{\lambda_{i}\right\}, 0 \leqslant \lambda_{i} \leqslant 1, \sum_{i=1}^{N} \lambda_{i}=1\right)$. All states $\rho$ are generated according to the Zyczkowski, Horodecki, Sanpera, and Lewenstein (ZHSL) [14] measure $\nu \times \mathcal{L}_{N-1}$. Here, $\nu$ is the measure induced on $\mathcal{P}$ by the Haar measure on the group of unitary matrices $U(N)$ and $\mathcal{L}_{N-1}$ is the Leguesbe measure on the simplex of eigenvalues $\Delta[25,26]$.

As stated above, we deal in this paper with two kinds of maximally entangled states (MEMS, and Ishizaka and Hiroshima ones). We call the class that comprises both kinds the ME one. Figure 1 depicts the overall situation. In the upper part we plot the ME-states' concurrence $C_{I H}$ versus. the participation ratio. $R$ ranges in the interval $1<R<1.8$ (the latter figure corresponds to the above-mentioned transition point for MEMS). (a) The upper line gives MEMS states and the inferior one the lower bound (7). (b) The lower part of the figure gives the conditional entropy of the ME states $S_{q}^{R}(A \mid B)$ for $q \rightarrow \infty$ (the solid curve corresponds to the MEMS case). It is always negative, so that here the entropic inequalities provide the correct answer in order to detect entanglement.

Figure 2 is a plot of the concurrence $C_{I H}$ vs $\lambda_{\max }$, the maximum eigenvalue of our ME bipartite states $\rho$. The dashed line corresponds to MEMS. The graph confirms the statement made in [15] that the latter are not maximally entangled states if mixedness is measured according to a criterion that is not the $R$ one. Three separate regions (I, II, III) 


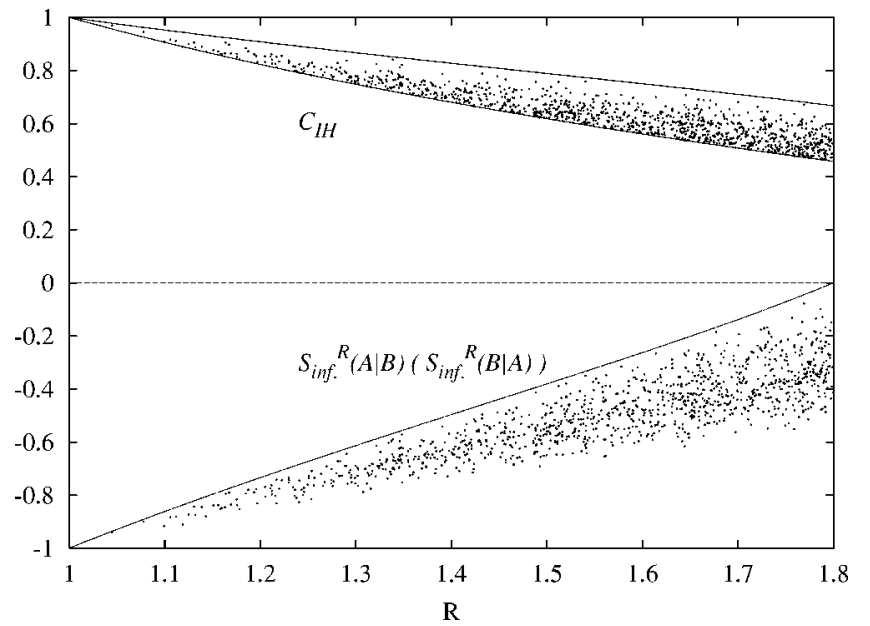

FIG. 1. Plot of the concurrence $C_{I H}$ for two kinds of maximally entangled states: Ishizaka and Hiroshima states (dots) and MEMS vs $R$ (upper solid curve) for a sample set. Their corresponding $S_{\infty}^{R}(A \mid B)$ values are also shown. Contour lines can be found analytically. See text for details.

can be seen to emerge. The maximum and minimum (continuous) contour lines are of an analytical character as seen in the following:

I. First zone:

(a) $C_{I H}^{\max }=\lambda_{\max }$ for $\lambda_{\max } \in[1 / 2,1]$,

(b) $C_{I H}^{\min }=2 \lambda_{\max }-1$ for Bell diagonal states.

II. Second zone:

(a) $C_{I H}^{\max }=3 \lambda_{\max }-1$ for $\lambda_{\max } \in[1 / 3,1 / 2]$,

(b) $C_{I H}^{\text {min }}=0$.

III. Third zone: all states are separable $C_{I H}=0$.

Our three zones (I, II, III) can be characterized according to strict geometrical criteria, as extensively discussed in [27]. In point of fact, the paper by Wei et al. [22] exhaustively studies MEMS for different measures of entanglement and

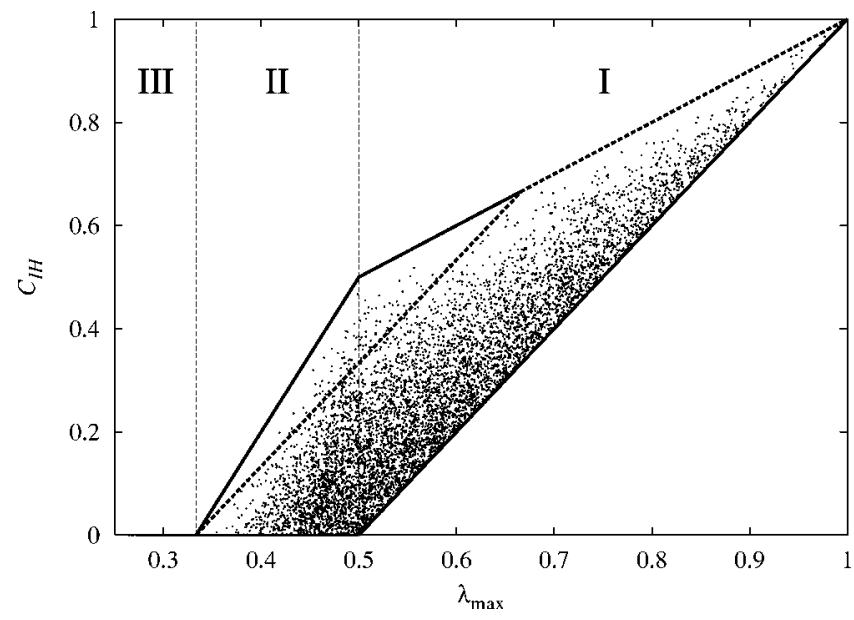

FIG. 2. Plot of the concurrence $C_{I H}$ for the class of maximally entangled states vs their maximum eigenvalue $\lambda_{\max }$ for a sample set of states. The dashed line corresponds to $\rho_{M E M S}$ states. Notice the fact that these states are not maximally entangled if the mixedness is not given by $R$. Maximum and minimum contour lines for $C_{I H}$ are found in analytical fashion. See text for details.

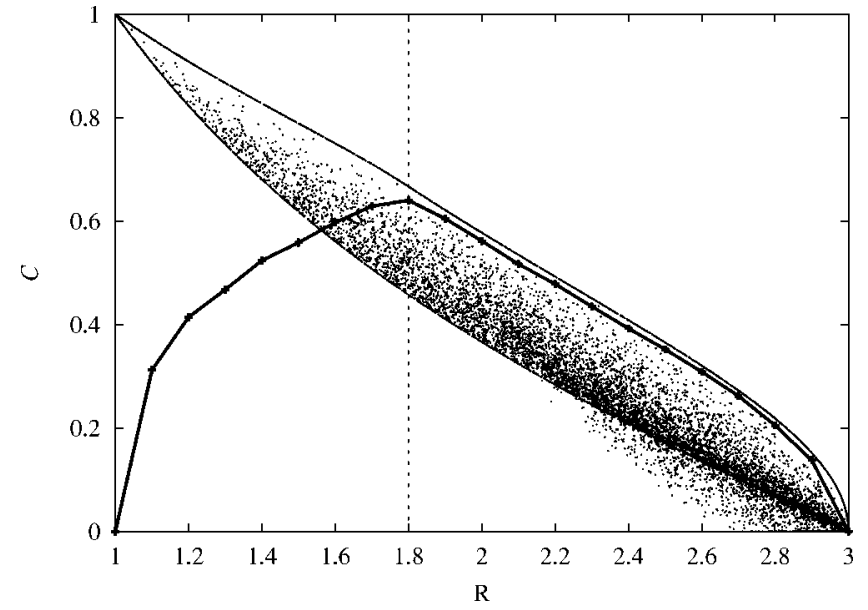

FIG. 3. Same as in Fig. 1, but for an extended $R$ range. The lower curve (with crosses on it) represents, for each $R$ value, the maximum concurrence for those states that obey classical entropic inequalities. The curve exhibits a maximum at $R=1.8$ and it vanishes at $R=1$ and $R=3$, where the entropic criterion is necessary and sufficient. That this curve does not exactly match the MEMS quasidiagonal curve above it, for the range $[1.8,3)$, is due to the relative scarcity of the pertinent states (generated randomly according to the ZHSL measure). See text for details.

mixedness. The extension made here to $\lambda_{\max }$ as a proper degree of mixture confirms in Fig. 2 the discussion given in [22] that asserts that MEMS are sensitive to the form of mixture employed.

Figure 3 is a $C_{I H}$ vs $R$ plot similar to that of Fig. 1, but for an extended $R$ range $(1<R<3)$. The pertinent IH bipartite states fill a "band" with dots (a sample of $10^{4}$ states). In Fig. 3 we focus attention on a special type of bipartite states: those that, being entangled, do fulfill the inequalities (4). For these states, let us call them entangled states with "classical" conditional entropic behavior (ESCRE), the quasitriangular solid line depicts, for each $R$, the maximum degree of entanglement attainable. For each value of $R$ (crosses), we generate $10^{8}$ states according to the aforementioned ZHSL measure, keeping only the ESCRE ones with maximal $C$. Interestingly enough, the maximum degree of entanglement for ESCRE obtains at $R=1.8$, which signals the change of regime for MEMS [cf. (5) and commentaries immediately below that equation]. This fact gives an entropic meaning to that particular $R$ value. We can state then that (i) whenever the entropic criterium turns out to constitute a necessary and sufficient condition for separability (at $R=1$ and $R=3$ ), the ESCRE degree of entanglement is null, and (ii) the ESCRE degree of entanglement is maximal at the Munro et al. change-of-regime $R$ value of 1.8 .

\section{CONCLUSIONS}

For entangled states with classical conditional entropic behavior (ESCRE), the maximum degree of entanglement attainable obtains at $R=1.8$. Even though the entropic criteria are not universally valid for all two-qubits states (yielding only a necessary condition for separability), they have been 
shown here to preserve their full applicability for an important family of states, namely, those states for which their entanglement cannot increase under the action of logic gates for participation rations in the interval $(R \in[1,1.8])$. This, in turn, gives an entropic meaning to this special $R$ value encountered by Munro et al. [15]. We find explicit boundaries to $C_{I H}$ when we express the degree of mixture using the maximum eigenvalue $\lambda_{\max }$ of $\rho^{I H}$. It would seem that the characterization of the entanglement for these states, using the $\lambda_{\max }$ criterion, provides the best insight into the entanglement features of these states. Beyond a certain value of the concurrence, all states, not necessarily the ones considered before, can be correctly described by the entropic inequalities as far as this criterion is concerned. One may argue that if the quantum correlations are strong enough (greater than $C_{R=1.8}^{\max }$ or $C_{\lambda_{\max }=2 / 3}^{\max }$ ), there is still room for entropic-based separability criteria to hold.

\section{ACKNOWLEDGMENTS}

This work was partially supported by the MEC Grant No. BFM2002-03241 (Spain) and FEDER (EU), by the Government of Balearic Islands, and by CONICET (Argentine Agency).
[1] H.-K. Lo, S. Popescu, edited by T. Spiller, Introduction to Quantum Computation and Information (World Scientific, River Edge, NJ, 1998).

[2] Quantum Computing and Quantum Communications, edited by C. P. Williams (Springer, Berlin, 1998).

[3] The Physics of Quantum Information, edited by D. Bouwmeester, A. Ekert, A. Zeilinger (Springer, Berlin, 1998).

[4] A. Ekert, Phys. Rev. Lett. 67, 661 (1991).

[5] C. H. Bennett, G. Brassard, C. Crepeau, R. Jozsa, A. Peres, and W. K. Wootters, Phys. Rev. Lett. 70, 1895 (1993).

[6] C. H. Bennett and S. J. Wiesner, Phys. Rev. Lett. 69, 2881 (1993).

[7] G. P. Berman, G. D. Doolen, R. Mainieri, and V. I. Tsifrinovich, Introduction to Quantum Computers (World Scientific, Singapore, 1998).

[8] R. F. Werner, Phys. Rev. A 40, 4277 (1989).

[9] A. Peres, Quantum Theory: Concepts and Methods (Kluwer, Dordrecht, 1993).

[10] C. H. Bennett, D. P. DiVicenzo, J. Smolin, and W. K. Wootters, Phys. Rev. A 54, 3824 (1996).

[11] W. K. Wootters, Phys. Rev. Lett. 80, 2245 (1998).

[12] J. Grondalski, D. M. Etlinger, and D. F. V. James, Phys. Lett. A 300, 573 (2002).

[13] P. Zanardi, C. Zalka, and L. Faoro, Phys. Rev. A 62, 030301 (2000).

[14] K. Zyczkowski, P. Horodecki, A. Sanpera, and M. Lewenstein, Phys. Rev. A 58, 883 (1998).
[15] W. J. Munro, D. F. V. James, A. G. White, and P. G. Kwiat, Phys. Rev. A 64, 030302 (2001).

[16] M. Casas, S. Martínez, F. Pennini, and A. Plastino, Physica A 305, 41 (2002).

[17] M. Gell-Mann and C. Tsallis, Nonextensive Entropy - Interdisciplinary Applications (Oxford University Press, Oxford, 2003).

[18] C. Tsallis, S. Lloyd, and M. Baranger, Phys. Rev. A 63, 042104 (2001).

[19] C. Tsallis, P. W. Lamberti, and D. Prato, Physica A 295, 158 (2001).

[20] J. Batle, A. R. Plastino, M. Casas, and A. Plastino, Eur. Phys. J. B 35, 391 (2003).

[21] S. Ishizaka, and T. Hiroshima, Phys. Rev. A 62, 022310 (2000).

[22] Tzu-Chieh Wei, K. Nemoto, P. M. Goldbart, P. G. Kwiat, W. J. Munro, and F. Verstraete, Phys. Rev. A 67, 022110 (2003).

[23] N. A. Peters, J. B. Altepeter, D. Branning, E. R. Jeffrey, TzuChieh Wei, and P. G. Kwiat, Phys. Rev. Lett. 92, 133601 (2004).

[24] M. Barbieri, F. De Martini, G. Di Nepi, and P. Mataloni, Phys. Rev. Lett. 92, 177901 (2004).

[25] K. Zyczkowski, Phys. Rev. A 60, 3496 (1999).

[26] J. Batle, M. Casas, A. R. Plastino, and A. Plastino, Phys. Lett. A 296, 251 (2002); 298, 301 (2002).

[27] J. Batle, M. Casas, A. R. Plastino, and A. Plastino, Phys. Lett. A 296, 251 (2002). 